Article

\title{
Analysis of Malfunctions in Selected Parking Systems in the Czech Republic
}

\author{
Simona Mikšíková ${ }^{1, *,+} \mathbb{0}$, David Ulčák ${ }^{2,+}$ (D) and František Kuda ${ }^{1}$ \\ 1 Department of Urban Engineering, Faculty of Civil Engineering, VŠB-Technical University of Ostrava, \\ 70800 Ostrava, Czech Republic; frantisek.kuda@vsb.cz \\ 2 Department of Applied Mathematics, Faculty of Electrical Engineering and Computer Science, \\ VŠB-Technical University of Ostrava, 70800 Ostrava, Czech Republic; david.ulcak@vsb.cz \\ * Correspondence: simona.miksikova@vsb.cz \\ + These authors contributed equally to this work.
}

Citation: Mikšíková, S.; Ulčák, D.; Kuda, F. Analysis of Malfunctions in Selected Parking Systems in the Czech Republic. Sustainability 2022, 14, 1826. https://doi.org/10.3390/ su14031826

Academic Editor: Laura Eboli

Received: 12 December 2021

Accepted: 1 February 2022

Published: 5 February 2022

Publisher's Note: MDPI stays neutral with regard to jurisdictional claims in published maps and institutional affiliations.

Copyright: (c) 2022 by the authors. Licensee MDPI, Basel, Switzerland. This article is an open access article distributed under the terms and conditions of the Creative Commons Attribution (CC BY) license (https:// creativecommons.org/licenses/by/ $4.0 /)$.

\begin{abstract}
The underlying cause of the problem with parking is the imbalance between demand and supply in key areas where demand for parking is high, but parking is limited due to excessive land prices. Newly-developed automated multi-storey parking facilities are able to automatically pick up and place cars on different storeys, with minimal requirements on space. In the paper we concentrate on Automated Parking Systems and their malfunction rate. Specifically, we analyzed two automated systems located in the Czech Republic, in the towns of Brno and Slaný. We employed time series and various testing hypotheses to compare the malfunction rate of the systems, and used information from practice. We conclude with an evaluation and a brief description of the optimization of systems and the use of innovative tools.
\end{abstract}

Keywords: engineering; management; automated parking systems; malfunction; correlation

\section{Introduction}

As the number of vehicles rises, there is the need to build infrastructure which meets heightened demands on capacity and car parks. The number of parked cars affects traffic safety and sustainability [1]. The problem with parking significantly worsens the experience of urban car users given that they are forced to spend longer periods traveling to available parking spaces, such requirements are further aggravated by surface transport, which is already overloaded in town centers, subsequently threatening the travel experience of all drivers at peak times. As various sources from 1927 to 2001 have stated, between 8 and $74 \%$ of urban traffic travels for parking [2]. A parking fee might alleviate the problem with parking to some extent, but this results in excessive costs for car users in certain districts having significant parking needs [3]. Sufficient parking facilities must be built in the years to come in order to bridge the gap between providing parking space and the demands on parking, particularly in central areas. The idea of automated, multi-storey facilities that put the limited space to better use has been proposed, in light of newly-developed automatic technology. In contrast to traditional parking facilities that require space for access roads, access lanes, and landscaping not involving parking space, automated elevators are used to take in and place cars, meaning that no other space is required for the transportation of cars and drivers, a fact which we can exploit to significantly reduce the use of land. The space required to allow car doors to be opened becomes unnecessary for every parking space, further reducing the average space used per car [3,4]. As a result of its compact, multi-storey structure, an automated parking system can significantly increase the number of parking spaces, and in doing so reduce the cost of land per car. When the driver attempts to park, he/she must first stop the car on the elevator, get out, and lock the vehicle. After receiving confirmation from the driver, the elevator automatically places the car in free 
space. To collect the car, the driver first sends a request, and the elevator then transports the vehicle back to the driver at ground level [5].

Parking facilities belong to the critical part of the infrastructure in terms of traffic systems and area reach [6]. Christiansen states that parking policy significantly affects traffic since most of the personal rides start and end on parking facility [7]. According to Wang et al., problems with parking facilities cause not only discomfort to citizens, everyday pedestrians, and local inhabitants, but also a negative impression of the town/city in question [8].

Prediction of parking slots demand can help dynamically adjust parking services to improve overall efficiency and has potential for optimization of parking slot searching process [9]. Thanks to advances in Information and Communication Technologies (ICT), we have access to a much more extensive portion of more detailed data on parking, allowing us to monitor the behavior of parking demand, malfunctions, etc., namely as time series of gathered data. Many studies, on the other hand, suggest a significant spatial correlation to travelers' behavior $[10,11]$. Also, unexpected factors can derail many models which are based solely on time factors, as we can see with the current COVID-19 pandemic [12].

In this study, we focused on Automated Parking Systems (APS) and their malfunction rate. Our long-term goal is to employ advanced statistical methods to analyze and propose optimization for APSs maintenance and structure since this kind of research has not been done extensively concerning APSs (see the next section). At the beginning of this study, we examined two APSs from the perspective of typology, and we subsequently moved ahead with the research itself based on data obtained from parking system operators. We analyzed the data as time series and evaluated them using various tests (e.g., Ljung-Box test, Mann-Whitney test, etc.). Finally, we evaluated the data, compared malfunctions, and looked in more detail at the individual systems and the causes of malfunctions.

\section{Literature Review}

In this section, we have a brief look at related works with an emphasis on time series. As far as it is known, there are no works that focus on malfunctions of APSs. Considering other parking-related works using statistical methods, there have been several methods for estimation of occupancy of parking slots in development. These methods can be divided into two categories: Statistical and Machine Learning methods, and Deep Neural Networks [13].

The first category focuses on using historical data to predict short-term occupancy, see e.g., [14]. Ziat et al. [15] method based on learning from the representative dataset for predicting actual workload and occupancy. This approach improved the reliability of predictions compared to purely statistical methods since it took into account also external influences, e.g., weather, traffic jams, etc., which can affect customer behavior regarding parking.

Most of the former studies did not take into account possible correlation among parking sites, which can also affect the behavior of drivers and thus warp the prediction [10]. Some of the aforementioned approaches are yet to be adapted for APSs specifically, giving subject to future work.

Although not directly related to APSs, there are similar works regarding park-and-ride facilities made, see [16,17].

\section{Materials and Methods}

In this section, we will get acquainted with both parking systems being compared. Both APSs belong to the MULTITOWER family, which is one of the two main reasons why we chose specifically these APSs. The other reason is the willingness of the practitioners to provide the necessary data which is, unfortunately, quite scarce.

\subsection{Automated Parking System in Slaný}

This car park with an Automated Parking System that facilitates short-term and longterm parking in the centre of the town was put into service in 2013. The car park building is located on the corner of Třebízského Street and Palackého Street. The site is located 
near the historic centre of the town of Slaný (Czech Republic) and is within the monument zone. Architecturally, the car park is incorporated into the existing urban structure. An automated loading facility is used to load and stack passenger cars. Cars are stacked on steel pallets which allow water or snow to drip off. The stacking spaces have natural ventilation and are not heated. The technological part of the loading system consists of pallets, platforms, and one lifting device. The loading system has a total capacity of 149 spaces. The facility is divided into 7 storeys, with the first storey at ground level, used for loading and unloading cars. The first underground storey and the 2nd storey contain 35 parking berths and 2 moving platforms, while the 3rd storey has 21 parking berths and 2 moving platforms. The 4th, 5th and 6th storey each hold 20 parking berths and 2 moving platforms. The storeys are connected by a lifting device fitted with a twin-pallet system.

\subsection{Automated Parking System without Platform in Brno}

The City of Brno owns an Automated Parking System, which is located in Kopečná Street right in the centre of the city. The system was put into service in 2014 and holds 88 parking spaces. The automated parking facility is used to load and stack passenger cars. The technological part of the loading system consists of pallets, turntables, and a lifting device. The facility is divided into 7 storeys. The first storey is at ground level and is predominantly used for parking and retrieving cars (loading and unloading bay with turntable), contains 4 parking berths, 2 berths in Section 2 and two berths in Section 3. There are also three lifting devices on this storey, a separate lifting device for each section. The other 6 storeys are used for parking cars, with each parking storey holding 15 parking berths. The storeys in each section of the car park are connected by a separate lifting device which is fitted with a platform that handles horizontal and vertical movements when parking and retrieving pallets with cars. The lifting device is designed in such a way that facilitates the replacement of one pallet with another. This means that the car that is being parked or retrieved is placed on the upper level of the lifting device, while the lower level of the lifting device holds a free pallet that immediately replaces the freed-up space to ensure that another vehicle can be parked there.

\subsection{Categorization of Possible Malfunctions}

The malfunctions identified in the bearing steel and machine structures of technological parts can generally be divided into 3 basic categories according to the degree of risk to the safety of the structure, operation, or workers.

1. Posing an immediate threat to the safety and reliability of the structure, the risk of breach of the structure, parts, and joints, and local or overall loss of stability. The malfunctions in this category include significant weakening of the steel bearing sections caused by the corrosive decay of material, breached joints and connections, amateurish modifications, or removal of parts of the structure, etc., which can initiate the partial or complete breakdown of the structure. In the case of such malfunctions to steel structures, repairs must be carried out immediately in order not to endanger the safety of persons in the building and the near vicinity.

2. Structures can perform their function, but their condition does not reliably guarantee safe operation, they are usually permanently and visibly deformed from overloading, the weakening of steel bearing sections by corrosive decay of material, reduced load-bearing capacity of the structure due to material fatigue, deformation of the structure as a whole as a result of subsidence, etc. In this case, we usually recommend a static evaluation on actual operating and climate loads and carrying out repairs or reconstruction on the closest possible date for periodic repairs. In case these malfunctions are not removed, a limiting factor is created which significantly reduces further safe and reliable operation of the building under consideration.

3. The structures can safely perform their function, but it is necessary to partially or fully restore their coatings, or occasionally repair or reconstruct the structures according to recommendations. 


\subsection{Input Data}

Analysis of malfunctions in Slaný-Input data were taken from a record of the monthly number of malfunctions at the car park in Slaný for the years 2017, 2018, and 2019.

Analysis of malfunctions in Brno-A data set of the malfunction rate of the system was provided by the practitioner, specifying the number of malfunctions in retrieving and parking and other malfunctions for the years 2017, 2018, and 2019. The system is divided into 3 sections, where the actual number of malfunctions is assigned to each section by month.

\section{Methods}

We will analyze data on malfunctions in Slaný and Brno as time series. First of all, let us consider the possible influence of our data on itself. In other words, how do the samples in our time series correlate with each other? This can be interpreted in practice as a repetitive type of malfunction, a malfunction that can cause other ones or seasonal influences (e.g., weather, holidays, etc.) for malfunction occurrence. One way of assessing this problem is to use an autocorrelation function $(A C F)$. This function evaluates Pearson's correlation coefficient for time series and displaced (lagged) version itself. This lag is the variable of $A C F$. It is clear that $A C F(0)=1$ because the correlation of the same data sets is maximum possible. In general, the value $A C F(k)$ speaks of the correlation between moments in time series, concerning samples remote from each other, i.e., in our case concerning months. If $A C F(k)$ differs significantly from 0 , this indicates an influence between sample $t$ and sample $t+k$. A significant $A C F$ may partly point to certain seasonal influences, as well as to the periods during which a certain trend is maintained. More formally, $A C F$ is defined as

$$
A C F(k)=\frac{1}{s^{2}(n-k)} \sum_{t=1}^{n}\left(X_{t}-\bar{X}\right)\left(X_{t+k}-\bar{X}\right),
$$

where $n$ is the number of samples, $X_{i}$ is the $i$-th data sample, $s$ is the sample standard deviation, $\bar{X}$ is the average of all values of the time series, and $k$ is the displacement (lag). The value of $A C F$ might also take into account the possible influence of the samples between $X_{t}$ and $X_{t+k}$; it necessarily does not need to be a direct influence. On the other hand, the partial autocorrelation function $(P A C F)$, is handier for describing this direct influence.

Before we proceed with a direct comparison of both time series, let us look at them individually.

As we can see in Figures 1 and 2, the data from Brno APS show a certain degree of correlation only for close consecutive months; see the $A C F$ and $P A C F$ values for lag $=1$. Specifically, the $P A C F$ value for the displacement of one month is 0.479 , which indicates a slight to moderate level of correlation. This can be interpreted in such a way that if there were a higher number of malfunctions in Brno in the given month, this could sometimes cause higher malfunction occurrence also in the following month, and vice versa, a low level of malfunctions with some probability indicated a low level of malfunctions in the following month. In contrast to this, it appears that in Slaný there are no significant influences of the data among one another; the $A C F$ and $P A C F$ values are relatively negligible; on the other hand, they are generally higher for values other than lag $=1$ than in the case of Brno. To evaluate the situation analytically, we use the Ljung-Box test (see [18]), which tests the hypothesis of zero autocorrelation across a time series. At significance level 0.05 , this test actually rejected the zero hypothesis for lag $=1$ in Brno $(p$-value $=0.004)$; autocorrelation here is therefore significantly nonzero; while in Slaný the conclusion is borderline ( $p$-value $=0.087)$. For higher lag values, however, the test no longer rejected the zero hypothesis, namely for lag $=12$ (due to possible seasonality), $p$-values came out as 0.376 and 0.511 , meaning that seasonal influences can be more or less excluded. As a result, we can say that autocorrelation influences are negligible for both time series, the only exception here and there being neighboring months in the data from Brno. 
Similar to $A C F$, the cross-correlation function $(C C F)$ measures the correlation for a certain value of lag, but this time between both time series. As seen in Figure 3, in our case CCF points to a moderate degree of correlation between data in Brno and Slaný. At the same time, it also shows a moderate degree of correlation for about half a year of the "lagged" Brno series with the series in Slaný.

We can use the Mann-Whitney test (for detail see [19]) to compare the values as a whole. This test examines whether there are significant differences between data, but does not take into account their nature as time series. At significance level 0.05 , this test did not reject the zero hypothesis ( $p$-value $=0.648$ ); the data do not, therefore, differ significantly, respectively the number of malfunctions in Brno and Slaný were more or less the same in the reference period. On the other hand, this does not mean the same structure.

Brno

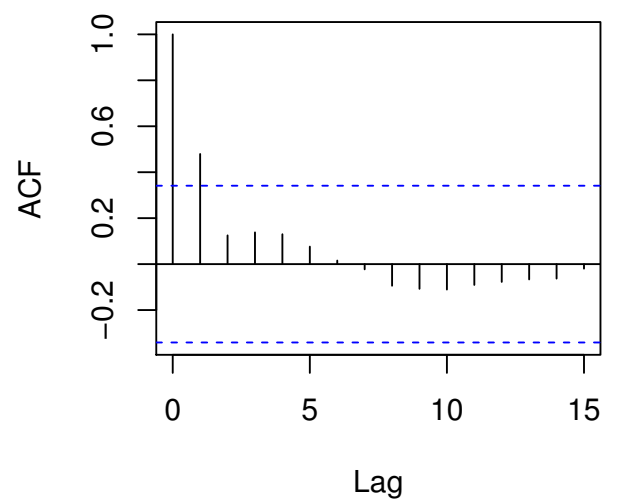

Slaný

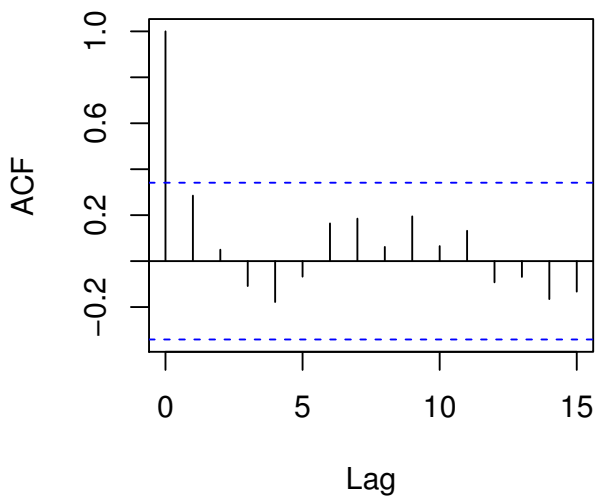

Figure 1. Autocorrelation graphs for number of malfunctions.

Brno

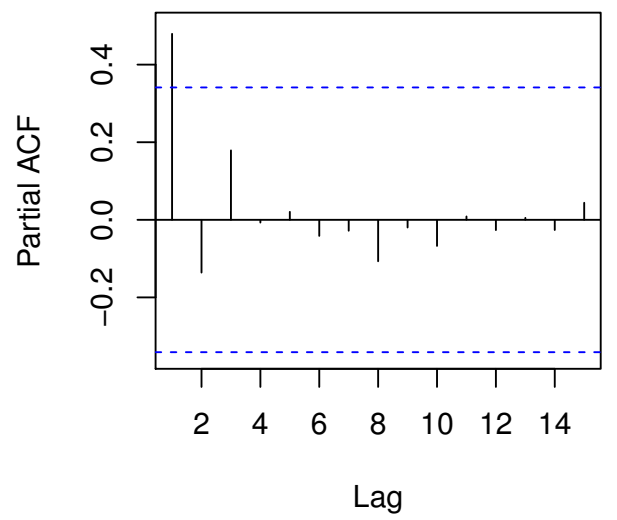

Slaný

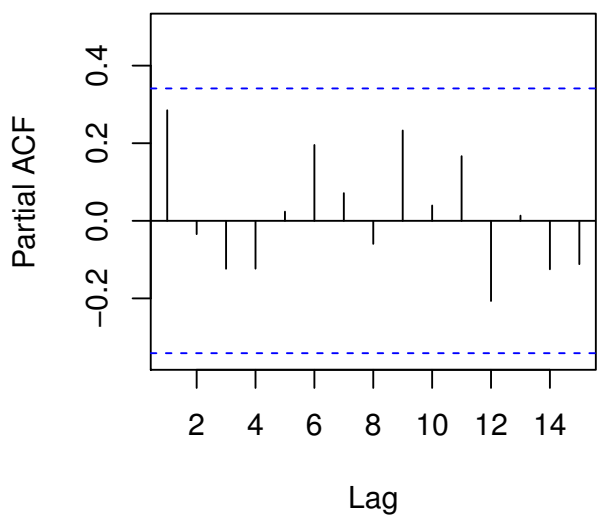

Figure 2. Partial autocorrelation graphs for number of malfunctions.

To compare the structural similarity of both data sets, we can use the Granger causality test, which evaluates whether it is possible to predict one time series using the values of another, while the zero hypothesis states that it is not. This causality does not need to be symmetric, i.e., the order of pair matters. For details about Granger causality, see [20]. In our case, we did not reject the zero hypothesis for both possible orders ( $p$-value $=0.499$ for pair B, S; $p$-value $=0.653$ for S, B). Hence, the structure of the data does not facilitate reciprocal prediction. Thus, the time series are structurally different, meaning that while not different in the number of malfunctions, both APSs suffered from the different intensity and types of malfunctions during the monitored period. To estimate the exact form of the difference, we can take a look at the trends of both series. 


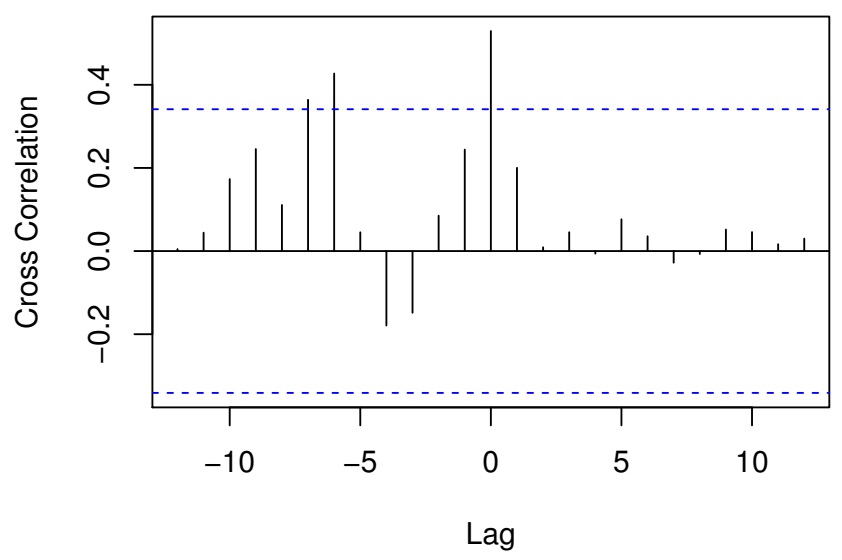

Figure 3. Cross-corelation of data in Brno and Slaný.

Figure 4 shows that both time series have an approximately similar trend. For better comparison, we can capture the trend with moving averages, for details see [21]. Let us compare the trends of both series using moving averages with exponential weighting (more recent data have greater weight):

As Figure 5 shows, the trend is similar in both cases-decreasing-although we can see that it has more or less stagnated in Slaný from the beginning of 2019, while a downward trend can still be seen in Brno. Similarly, a more pronounced decrease can be observed from linear regression, i.e., approximation of data in form of $Y \sim \alpha X+\beta$ in the sense of quadratic norm. The linear component $\alpha$ for Brno data equals -0.679 , whereas in Slaný it is only -0.347 , nodding towards a more significant decrease in Brno. This is, among other possible causes, due to a different dominant type of malfunctions in both APSs, see the following section.

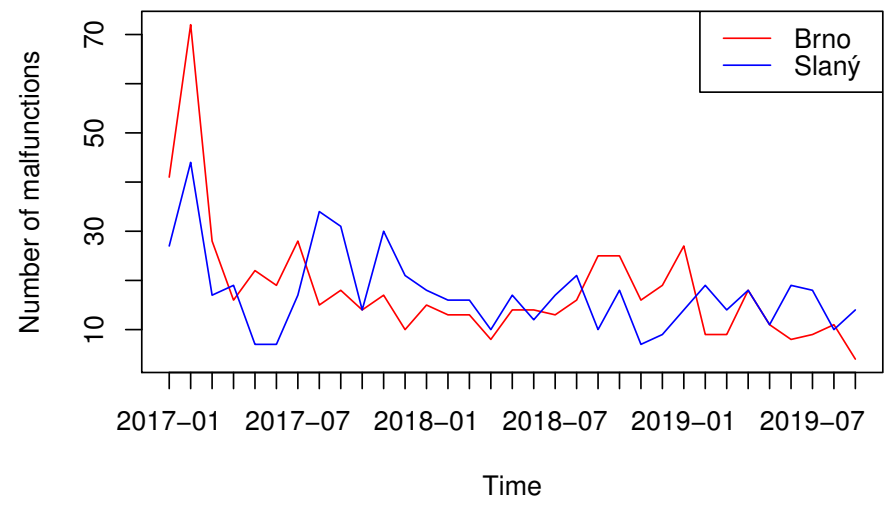

Figure 4. Depiction of the data within both time series.

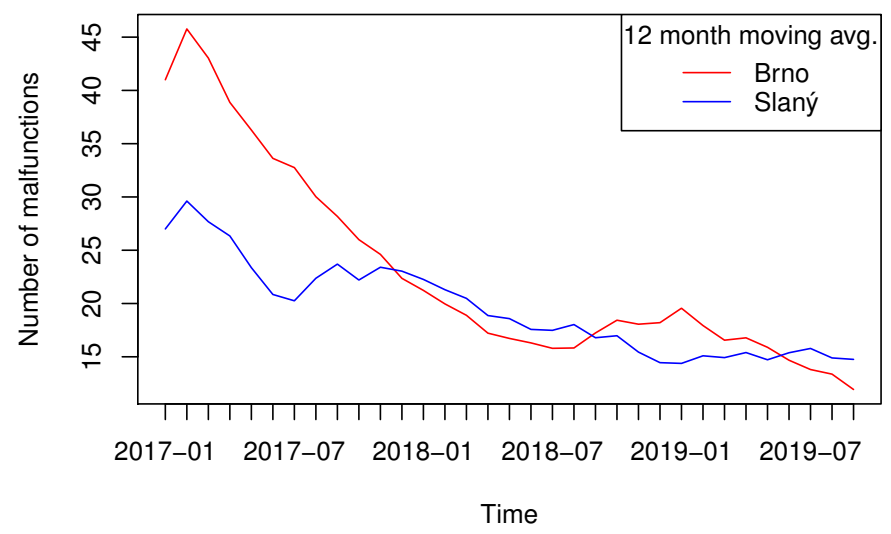

Figure 5. Approximated trends of both time series. 


\section{Results}

According to data testing, we found that the number of malfunctions is comparable in both, but that the structure of malfunctions differs and that Brno shows a more sustained decrease in the number of malfunctions. According to Tables 1 and 2, we can see, that the number of malfunctions is comparable in both APSs and likely decreasing in Brno, despite Brno having much more workload than APS in Slaný-the cars-per-month/capacity ratio in Brno is more than twice as large as the one in Slaný. Also, according to the results of the Granger test stated in the previous section, we can see that structure and type of malfunctions in both APSs differ. Considering overall better working performance in Brno (taking larger workload into account) we can conclude that-during the monitored period-malfunctions in Brno were more frequently of the second and especially the third type (as described in Section 3.3) than in Slaný, nodding towards higher reliability and possible profit of APS in Brno.

Table 1. Some basic parameters of APS in Slaný.

\begin{tabular}{lc}
\hline Parameter & Value \\
\hline Capacity & 149 \\
Avg. cars per month & 887 \\
\hline Max. mass of car [kg] & 3000 \\
Max. length of car [mm] & 5300 \\
Max. width of car [mm] & 2200 \\
Max. height of car [mm] & 1900 \\
\hline
\end{tabular}

Table 2. Some basic parameters of APS in Brno.

\begin{tabular}{lc}
\hline Parameter & Value \\
\hline Capacity & 88 \\
Avg. cars per month & 1126 \\
\hline Max. mass of car [kg] & 3000 \\
Max. length of car [mm] & 5400 \\
Max. width of car [mm] & 2200 \\
Max. height of car [mm] & 1900 \\
\hline
\end{tabular}

Let us look at the malfunctions in a little more detail. The most frequent malfunctions in the MULTITOWER system in Slaný are exceeding the maximum retrieval time and exceeding the maximum parking time. The reason for the maximum retrieval time is, for example, that the platform loads the pallet from the berth empty, meaning before the carrier it does not pick the pallet at all. This might be because the pallet was in the berth, a few millimeters behind the brakes, but such that the sensor detected the presence of the pallet. The method of correcting the cause of the malfunction is to clear the malfunction and load the pallet manually. Another possible cause of the malfunction is that the platform travel frequency inverter signals a malfunction that could not be cleared, which means that the system was unable to unhook the pallet, meaning that a technician has to put the pallet back into the berth and then load it again. There are also various malfunctions in the case of exceeding the maximum parking time; for example, the system has parked the pallet into a berth where it was already physically full, but one of the pallets was not entered in the visualization. Somewhere mistakenly deleted by operating staff in the past. The method of elimination involves the pallet being returned to the demalfunction position on the platform in manual mode, the missing pallet is then written into the berth, and the system then unloads the pallet into a free berth. The second example is that the pallets were not hooked when hooking, which leads to a collision of hooks. The method of elimination is such that the pallet is set in the berth by hand until properly hooked with the pallet on the platform. There are various malfunctions in the case of exceeding the maximum retrieval and parking time, and listing all causes and methods of elimination is not the 
subject matter of the paper. The exact numbers of retrieval and parking malfunctions in individual months are important, dominating the total number of malfunctions in the relevant month.

Parking malfunctions in the MULTITOWER system in Brno related to sensors, when they detected swerving pallets, and dripping water. Furthermore, malfunctions in the entrance door or incorrect unloading of pallets. Examples of retrieval malfunctions include the elevator unloading an empty pallet into a berth, the elevator unloading a pallet with a car into the berth but failing to print the pallet which was in the berth from the brakes, and many other malfunctions.

The companies that operate these buildings try to eliminate malfunctions to a minimum. Work is complicated, in that certain malfunctions occur quite randomly and cannot be monitored with $100 \%$ success. Malfunctions which affect the customer are unpleasant for an APS. These are malfunctions occurring at the time of retrieval when the customer has to wait for the elimination of a random malfunction. The customer does not experience any malfunctions when parking. The APS is automatic, but like any system may report malfunctions or error messages and an operator is, therefore, present to deal with these problems in situ.

\section{Discussion}

The purpose of this study was to examine selected Automated Parking Systems in terms of typology and malfunction rate. It is almost impossible to eliminate malfunctions. An APS is a highly-sophisticated system and machine that sometimes fails-heat factors, sensor failure, safety features, etc. In general, if the technology is properly designed as a whole, precisely assembled and set, the occurrence of malfunctions is minimal, assuming regular inspection, setting, lubrication, etc. At the same time, the driver must be able to operate the parking system.

Optimizing APS can be understood as the number of entry and exit areas per number of parked cars, which influences the most desired requirement-time, i.e., how long before another customer can park or how long it takes for a vehicle to be retrieved. Entry and exit are generally possible from one place, according to the specific situation, thus we furnish APS with turns that facilitate entry and exit head-on, without the need for reversing. Further optimization might be software optimization; i.e., at a time of technological standstill, the control system automatically moves cars from more distant positions closer to the exit area so that the time to wait for the vehicle to arrive is minimized. It is also possible to switch priorities between parking and retrieval according to the frequency of requests.

Various innovations need to be implemented to avoid malfunctions. Innovations from conceptual solutions (for example, from circular arrangement to rectangular-saving space), the development of new segments fixing pallets in a precise location, more precise storage of telescope guidance, software modifications which facilitate service interventions via remote connectivity, camera monitoring, etc. The biggest innovative element under preparation is the replacement of the existing loading technology with new vehicle handling using autonomous, intelligent trolleys, which will make it quicker to deal with clients and at the same time eliminates the disruption of APS operation in the event of a breakdown of one of the lifts.

Of course malfunctions can not be eliminated, regardless of the optimal capacity or type of APS, at the very least because of the human factor. For instance, some customers tend to ignore the instructions, e.g., they do not place the car in the check-in area properly, they do not break the car (parking gear in automatic cars is insufficient), they do not give proper instructions, they move around the check-in area even after the parking process beings, etc. On the other hand, these problems seem to be less of an issue for example in Warsaw, where there is an APS for a residential building. 


\section{Conclusions}

We see two different parking structures when comparing the Automated Parking Systems in Brno and Slaný. There is an APS with a capacity of 149 parking spaces in the town of Slaný and one with a capacity of 88 parking spaces in Brno. There is also a difference in the design: a multi-row MULTITOWER in Slaný and a MULTITOWER without a platform in Brno. There is also a significant difference in the average parking time of a passenger car: in Slaný a maximum of $95 \mathrm{~s}$ and in Brno $193 \mathrm{~s}$. The test of the hypothesis focused on an analysis of malfunctions in individual systems, the objective being to determine whether there are any statistically significant differences in the monthly number of malfunctions. Based on the evaluation, we found that the data did not differ significantly, but due to the difference in capacity and the maximum time taken to park a vehicle, it can be said that the system in Brno has a higher malfunction rate than the system in Slaný. The car park in Slaný has a negotiable "check-in" area, there is no need to turn the car, the elevator takes it from the side and a platform picks it up on the relevant storey, placing the car in a berth. The situation in Brno is different to save on space; here the car drives up to the elevator head-on and is then turned using a turntable. The elevator here also works as a platform-at the same time as lifting to the relevant storey, it places cars straight into the relevant berths. This makes the system simpler and more reliable in terms of malfunction rate, but unfortunately, this system was innovative and untested and therefore the malfunction rate may seem high. Those figures have now been radically reduced, mainly due to the experience of the previous setting. Furthermore, the number of malfunctions was shown to be significantly better, considering the larger workload of APS in Brno. More systems with smaller capacity rather than less large ones seem like the right solution for effective maintenance.

In the future, we would like to gather more detailed data and use other approaches, such as least square projection and confidence bounds for prediction of expenses, malfunctions, etc. We could employ also the machine learning approach, given suitable data, improving the number of factors considered and precision of predictions.

Author Contributions: Conceptualization, S.M.; methodology, S.M. and D.U.; software, D.U.; validation, F.K., S.M. and D.U.; formal analysis, D.U.; investigation, S.M.; resources, S.M.; data curation, D.U.; writing-original draft preparation, S.M. and D.U.; writing-review and editing, F.K. and S.M.; visualization, D.U.; supervision, F.K.; project administration, S.M.; funding acquisition, S.M. All authors have read and agreed to the published version of the manuscript.

Funding: This work was supported by the VŠB-TUO Student Grant Competition. The project Reg. No. are SP2021/102 and SP2021/95.

Institutional Review Board Statement: Not applicable.

Informed Consent Statement: Not applicable.

Data Availability Statement: The data were gathered from the practitioners of APSs in Brno and Slaný and are available from the authors on e-mail request.

Conflicts of Interest: The authors declare no conflict of interest.

\section{Abbreviations}

The following abbreviations are used in this manuscript:
APS
Automated parking system
ACF Auto-correlation function
PACF Partial auto-correlation function
CCF Cross-correlation function

\section{References}

1. Poliak, M.; Poliaková, A.; Čulík, K. Impact of the social law on truck parking sustainability in the EU. Sustainability 2020, $12,9430$. [CrossRef]

2. Shoup, D.C. Cruising for parking. Transp. Policy 2006, 13, 479-486. [CrossRef] 
3. Wang, Y.; Li, M.; Lin, X.; He, F. Online operations strategies for automated multistory parking facilities. Transp. Res. Part E Logist. Transp. Rev. 2021, 145, 102135. [CrossRef]

4. Nourinejad, M.; Bahrami, S.; Roorda, M.J. Designing parking facilities for autonomous vehicles. Transp. Res. Part B Methodol. 2018, 109, 110-127. [CrossRef]

5. Patrascu, D. How Automated Parking Systems Work. Autoevolution. 2010. Available online: https://www.autoevolution.com/ news/how-automated-parking-systems-work-19523.html (accessed on 28 October 2021).

6. Rifai, A.I. Analysis of Impact COVID-19 on Parking Characteristics in the Office Area: Case of Jakarta City. In Proceedings of the International Conference on Industrial Engineering and Operations Management, Singapore, 7-11 March 2021; pp. $487-495$.

7. Christiansen, P.; Engebretsen, Ø.; Fearnley, N.; Hanssen, J.U. Parking facilities and the built environment: Impacts on travel behaviour. Transp. Res. Part A Policy Pract. 2017, 95, 198-206. [CrossRef]

8. Wang, Y.-L.; Wang, X.; Zhang, M.-C. Current situation and analysis of parking problem in Beijing. Procedia Eng. 2016, 137, 777-785.

9. Fiez, T.; Ratliff, L.J.; Dowling, C.; Zhang, B. Data driven spatio-temporal modeling of parking demand. In Proceedings of the 2018 Annual American Control Conference (ACC), Milwaukee, WI, USA, 27-29 June 2018; pp. 2757-2762.

10. Gong, S.; Mo, X.; Cao, R.; Liu, Y.; Tu, W.; Bai, R. Spatio-temporal Parking Behaviour Forecasting and Analysis Before and during COVID-19. arXiv 2021, arXiv:2108.07731.

11. Gong, S.; Cartlidge, J.; Bai, R.; Yue, Y.; Li, Q.; Qiu, G. Extracting activity patterns from taxi trajectory data: A two-layer framework using spatio-temporal clustering, Bayesian probability and Monte Carlo simulation. Int. J. Geogr. Inf. Sci. 2020, 34, 1210-1234. [CrossRef]

12. World Health Organization. Coronavirus Disease 2019 (COVID-19): Situation Report, 54; World Health Organization: Geneva, Switzerland, 2020.

13. Yang, S.; Ma, W.; Pi, X.; Qian, S. A deep learning approach to real-time parking occupancy prediction in transportation networks incorporating multiple spatio-temporal data sources. Transp. Res. Part C Emerg. Technol. 2019, 107, 248-265. [CrossRef]

14. Pullola, S.; Atrey, P.K.; El Saddik, A. Towards an intelligent GPS-based vehicle navigation system for finding street parking lots. In Proceedings of the 2007 IEEE International Conference on Signal Processing and Communications, Dubai, United Arab Emirates, 24-27 November 2007; pp. 1251-1254.

15. Ziat, A.; Leroy, B.; Baskiotis, N.; Denoyer, L. Joint prediction of road-traffic and parking occupancy over a city with representation learning. In Proceedings of the 2016 IEEE 19th International Conference on Intelligent Transportation Systems (ITSC), Rio de Janeiro, Brazil, 1-4 November 2016; pp. 725-730.

16. Ortega, J.; Tóth, J.; Péter, T.; Moslem, S. An integrated model of park-and-ride facilities for sustainable urban mobility. Sustainability 2020, 12, 4631. [CrossRef]

17. Ortega, J.; Moslem, S.; Palaguachi, J.; Ortega, M.; Campisi, T.; Torrisi, V. An integrated Multi Criteria Decision Making Model for evaluating park-and-ride facility location issue: A case study for Cuenca city in Ecuador. Sustainability 2021, 13, 7461. [CrossRef]

18. Brockwell, P.J.; Davis, R.A. Introduction to Time Series and Forecasting; Springer: New York, NY, USA, 2016.

19. Bonnini, S.; Corain, L.; Marozzi, M.; Salmaso, L. Nonparametric Hypothesis Testing: Rank and Permutation Methods with Applications in R; John Wiley \& Sons: Hoboken, NJ, USA, 2014.

20. Granger, C.W. Investigating causal relations by econometric models and cross-spectral methods. Econom. J. Econom. Soc. 1969, 37, 424-438. [CrossRef]

21. Holt, C.C. Forecasting seasonals and trends by exponentially weighted moving averages. Int. J. Forecast. 2004, 20, 5-10. [CrossRef] 